\title{
Polarization immunity of magnetoresistivity response under microwave excitation
}

\author{
Jesús Iñarrea ${ }^{1,2}$ and Gloria Platero ${ }^{2}$ \\ ${ }^{1}$ Escuela Politécnica Superior, Universidad Carlos III,Leganes, Madrid,Spain \\ ${ }^{2}$ Instituto de Ciencia de Materiales, CSIC, Cantoblanco,Madrid,28049,Spain.
}

(Dated: July 8, 2018)

\begin{abstract}
We analyze theoretically the dependence of the longitudinal magneto-resistivity response of microwave irradiated two-dimensional electron systems on the microwave polarization. Both linear and circular polarizations are considered. Recent experiments show that resistivity oscillations and zero resistance states are unaffected by changing the polarization of the microwave field. We propose a plausible explanation for the experimentally observed magneto-resistivity polarization immunity.

PACS numbers:
\end{abstract}

Recently, magneto-transport experiments on twodimensional electron systems(2DES) irradiated with microwaves have shown two very interesting features: Microwave Induced Resistivity Oscillations (MIRO) [1, 2] and Zero Resistance States (ZRS) [3, 4]. This has motivated intense activity, both experimental and theoretical. New and remarkable experimental contributions are being published on a continual basis. Among them, one can note the activated temperature dependence in the magneto-resisitivity $\left(\rho_{x x}\right)$ response 3, 4, 5], quenching of $\rho_{x x}$ at high microwave (MW) intensities [2, 6], absolute negative conductivity [2, 5, 6, 7], suppression of MIRO and ZRS by in-plane magnetic field [8, 9], and the behavior of $\rho_{x x}$ under bichromatic MW radiation coming from two monochromatic sources with different frequencies [10]. Possibly the observation that MIRO and ZRS are notably immune to the polarization of MW radiation in Ref[[11]] is one of the most surprising results. In this experiment the influence of the MW polarization on $\rho_{x x}$ in a $2 \mathrm{DES}$ was analyzed. Different MW polarizations were used, circular in both senses (left and right) and also linear in $\mathrm{x}$ (current direction) and $\mathrm{y}$ directions. The unexpected result of almost complete immunity of $\rho_{x x}$ with the polarization was obtained. All these new experimental results provide new and real challenges for the theoretical models presented to date [12, 13, 14, 15, 16, 17, 18, 19, 20, 21, 22].

Some theoretical contributions have been presented that can explain some of the new experimental outcomes. We can note proposals for explaining the behavior of the MW driven $\rho_{x x}$ with temperature and with high MW intensities 23, 24], the observed absolute negative conductivity [25, 26], and the $\rho_{x x}$ response to bichromatic MW radiation[27, 28]. Regarding MWpolarization immunity, while some theoretical models predict strikingly different dependences on the radiation polarization 14, 18, 20], in others only linear radiation was considered [16, 17, 19].

In this letter we propose a theoretical explanation for the experimental evidence which shows that $\rho_{x x}$ does not depend on the MW polarization. Our theoretical results are based on the driven Larmor orbits model[19]. In a recently presented work by the authors 19], it was shown that in a 2DES subjected to a moderate perpendicular magnetic field and MW radiation, the Larmor orbit centers oscillate back and forth in the $x$ direction with the same frequency as the MW field. A major and non-trivial extension of this model [19] is presented here which allows different polarizations for the MW field to be considered, namely elliptical, circular or linear.

The results presented in this letter can be applied and generalized to any physical situation consisting of a quantum mechanical oscillator excited by any time-dependent force. We can cite, for instance, nano-electromechanical systems (NEMS), molecular electronics, surface acoustic waves (SAW) in Hall bars, vibrational and rotational molecular spectra, etc. In the case of a harmonic timedependent force the physics obtained is even richer, because new resonance situations arise.

Our system consist in a 2DES subjected to MW radiation, that can be in different polarization states, a perpendicular magnetic field $(B)$ (z-direction) and a DC electric field $\left(E_{d c}\right)$ (x-direction). In order to introduce the model we consider initially left-circularly polarized MW, i.e., the electric field $\vec{E}(t)$ of the MW radiation is:

$$
\vec{E}(t)=E_{0}(\vec{i} \cos w t+\vec{j} \sin w t)
$$

(a detailed mathematical analysis for other polarizations will be presented elsewhere 29]). $E_{0}$ and $w$ are the amplitude and frequency of the MW field respectively. The total hamiltonian $H$, working with the symmetric gauge for the vector potential of $B:\left(\overrightarrow{A_{B}}=-\frac{1}{2} \vec{r} \times \vec{B}\right)$, can be written as:

$$
\begin{aligned}
& H= \frac{P_{x}^{2}+P_{y}^{2}}{2 m^{*}}+\frac{w_{c}}{2} L_{z}+\frac{1}{2} m^{*}\left[\frac{w_{c}}{2}\right]^{2}\left[(x-X)^{2}+y^{2}\right] \\
&-\frac{e^{2} E_{d c}^{2}}{2 m^{*}\left[\frac{w_{c}}{2}\right]^{2}}-e E_{0} \cos w t(x-X)-e E_{0} x \sin w t \\
&-e E_{0} \cos w t X \\
&= H_{1}-e E_{0} \cos w t X \\
& X= \frac{e E_{d c}}{m^{*}\left(w_{c} / 2\right)^{2}} \text { is the center of the orbit for the electron } \\
& \text { cycloidal motion, } e \text { is the electron charge, } E_{d c} \text { is the DC }
\end{aligned}
$$


electric field in the current direction, $w_{c}$ is the cyclotron frequency and $L_{z}$ is the z-component of the electron total angular momentum. $H_{1}$ can be solved exactly after lengthy algebra 29], and using this result allows an exact solution for the electronic wave function of $H$ to be obtained:

$$
\begin{aligned}
& \Psi(x, y, t)=\phi_{N}[(x-X-a(t)),(y-b(t)), t] \\
& \times \exp \frac{i}{\hbar}\left[m^{*}\left(\frac{d a(t)}{d t} x+\frac{d b(t)}{d t} y\right)+\frac{m^{*} w_{c}(b(t) x-a(t) y)}{2}-J_{0}\right. \\
& \times \sum_{p=-\infty}^{\infty} J_{p}\left(A_{N}\right) e^{i p w t}
\end{aligned}
$$

where $\phi_{N}$ are the analytical solutions for the Schrödinger equation with a two-dimensional (2D) parabolic confinement, known as Fock-Darwin states [30]. The FockDarwin states converge to a Landau level spectrum when $B$ is large or it is the only source of confinement (present case). In polar coordinates $\phi_{N}(r, \theta, t)$ can be expressed as:

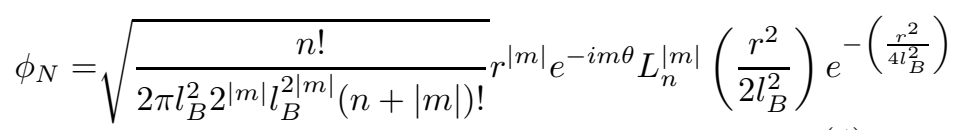

where $n$ is the radial quantum number, $m$ is the angular momentum quantum number, $L_{n}^{|m|}$ are the associated Laguerre polynomials and $l_{B}$ is the effective magnetic length. For the polar coordinates:

$r^{2}=[x-X-a(t)]^{2}+[y-b(t)]^{2}$ and

$r e^{i \theta}=[x-X-a(t)]+i[y-b(t)]$.

$a(t)$ (for the x-coordinate) and $b(t)$ (for the y-coordinate) are the solutions for a classical driven 2D harmonic oscillator (classical uniform circular motion). The expressions in the case of a left polarized MW radiation are:

$$
\begin{aligned}
& a(t)=\frac{e E_{o}}{m^{*} \sqrt{w^{2}\left(w_{c}-w\right)^{2}+\gamma^{4}}} \cos w t=A_{-} \cos w t \\
& b(t)=\frac{e E_{o}}{m^{*} \sqrt{w^{2}\left(w_{c}-w\right)^{2}+\gamma^{4}}} \sin w t=A_{-} \sin w t(5)
\end{aligned}
$$

$\gamma$ is a material and sample-dependent damping factor which dramatically affects the movement of the MWdriven electronic orbits, and which has been introduced phenomenologically. Along with this movement interactions occur between electrons and lattice ions, yielding acoustic phonons and producing a damping effect in the electronic motion. In Ref. 23], we developed a microscopical model to calculate $\gamma$, estimating a numerical value of $\gamma \simeq 10^{12} s^{-1}$ for GaAs. $L$ is the classical lagrangian:

$$
\begin{aligned}
L= & \frac{m}{2}\left[\left(\frac{d a(t)}{d t}\right)^{2}+\left(\frac{d b(t)}{d t}\right)^{2}\right] \\
& -m \frac{w_{c}}{2}\left[a(t) \frac{d b(t)}{d t}+b(t) \frac{d a(t)}{d t}\right]
\end{aligned}
$$

FIG. 1: Calculated magneto-resistivity $\rho_{x x}$ as a function of $B$ for circularly and linearly polarized radiation for the experimental frequencies used, 100,183 and 200GHz. In the case of circular light (left panels) the two senses of polarization have been considered: left, single line (black color online) and right, dotted line (red color one line) . For linear MW (right panels), we have considered the $\mathrm{x}$ direction, single line (black color online) and y direction, dotted line (red color online). The $\rho_{x x}$ polarization immunity can be observed clearly for the three frequencies, especially for $B$ below the cyclotron resonance (see vertical dashed line, blue color online). $\mathrm{T}=1 \mathrm{~K}$.

and $J_{p}$ are Bessel functions, whose arguments, $A_{N}$, are given by (for left circular MW):

$A_{N}=\frac{e E_{0}}{\hbar} X\left(\frac{1}{w}+\frac{w+w_{c}}{\sqrt{\left(w_{c}^{2}-w^{2}\right)^{2}+\gamma^{4}}}-\frac{w_{c}\left(w+w_{c}\right)}{2 w \sqrt{\left(w_{c}^{2}-w^{2}\right)^{2}+\gamma^{4}}}\right)$

Similar, but not identical, expressions for $\Psi(x, y, t)$ are obtained for right and linear ( $\mathrm{x}$ or $\mathrm{y}$ direction) polarized MW radiation [29]. The key differences among them are given by the expressions we obtain for $\mathrm{a}(\mathrm{t})$ and $\mathrm{b}(\mathrm{t})$. 
Thus, for instance, for right polarized MW light:

$$
\begin{aligned}
& a(t)=\frac{e E_{o}}{m^{*} \sqrt{w^{2}\left(w_{c}+w\right)^{2}+\gamma^{4}}} \cos w t=A_{+} \cos w t \\
& b(t)=\frac{-e E_{o}}{m^{*} \sqrt{w^{2}\left(w_{c}+w\right)^{2}+\gamma^{4}}} \sin w t=A_{+} \sin w t .
\end{aligned}
$$

The first important result we obtain is that, apart from phase factors, the wave function for $H$ is the same as a Fock-Darwin state where the center of the electron orbits performs a circular motion in the xy plane with frequency $w_{c}$, given by $a^{2}(t)+b^{2}(t)=A_{\mp}^{2}$, (" - " for the case of left circular MW and " + " for right circular light).

Electrons suffer scattering due to charged impurities that are randomly distributed in the sample. If the scattering is weak, we can apply time dependent first order perturbation theory. To proceed we calculate the impurity scattering transition rate $W_{N, M}$ from an initial state $\Psi_{N}(x, y, t)$, to a final state $\Psi_{M}(x, y, t)[19,31]$ :

$W_{N, M}=\lim _{\alpha \rightarrow 0} \frac{d}{d t}\left|\frac{1}{i \hbar} \int_{-\infty}^{t^{\prime}}<\Psi_{M}(x, t)\right| V_{s}\left|\Psi_{N}(x, t)>e^{\alpha t} d t\right|^{2}$

where $V_{s}$ is the scattering potential for charged impurities [32]. After some algebra we obtain for this transition rate $W_{N, M}$ :

$$
W_{N, M}=\alpha \frac{e^{5} n_{i} B S}{16 \pi^{2} \epsilon^{2} \hbar^{2}}\left[\frac{\Gamma}{\left[\hbar w_{c}(N-M)\right]^{2}+\Gamma^{2}}\right]
$$

$\Gamma$ is the Landau level broadening, $n_{i}$ is the impurity density, $S$ the surface of the sample and $\epsilon$ the dielectric constant. Since Coulomb interactions are not the primary focus of this work, we will use the dimensionless parameter $\alpha$ to control the strength of the electron-charged impurity interaction, rather than considering a more sophisticated functional form. With $\alpha=1$ representing the "bare" Coulomb interaction, using a screened scattering potential corresponds to $\alpha<133$.

The next step is to find the average effective distance advanced by the electron in every scattering jump which is given by (see Ref. [19] for a detailed explanation): $\Delta X^{M W}=\Delta X^{0}+A_{\mp} \cos w \tau$, where $\Delta X^{0}$ is the effective distance advanced when there is no MW field present and $1 / \tau=W_{N, M}$ ( $\tau$ being the impurity scattering time). The magnitude $A_{\mp}$ is the amplitude of the orbit center motion in the $\mathrm{x}$-direction $(\mathrm{a}(\mathrm{t}))$ :

$$
A_{\mp}=\frac{e E_{o}}{m^{*} \sqrt{w^{2}\left(w_{c} \mp w\right)^{2}+\gamma^{4}}}
$$

Considering that we have a static electric field $E_{d c}$ in the current direction (x direction), we obtain an average value (over all the scattering processes) for $\triangle X^{M W}$ different from zero in that direction. Therefore the electron possesses an average drift velocity $v_{N, M}$ in the $\mathrm{x}$ direction. This drift velocity can be readily calculated by introducing the term $\triangle X^{M W}$ into the expression of the transition rate $W_{N, M}$, and finally the longitudinal (or diagonal) conductivity $\sigma_{x x}$ can be obtained [19, 31]. To calculate $\rho_{x x}$ we use the relation $\rho_{x x}=\frac{\sigma_{x x}}{\sigma_{x x}^{2}+\sigma_{x y}^{2}}$, where $\sigma_{x y} \simeq \frac{n_{i} e}{B}$ and $\sigma_{x x} \ll \sigma_{x y}$.

All our results have been based on parameters corresponding to experiments by Smet et al. 11]. In Fig.1 we show $\rho_{x x}$ obtained using our model as a function of $B$ for circularly and linearly polarized radiation and for the experimental frequencies. In the case of circular light (left panels) the two senses of polarization have been considered. For linear MW we have considered the $\mathrm{x}$ and $\mathrm{y}$ direction (right panels). As in experiments, the calculated $\rho_{x x}$ response is practically immune to the polarization sense of circularly polarized MW radiation, specially for $B$ below cyclotron resonance (see vertical dashed line). We obtain similar results in terms of oscillations and ZRS for the different polarizations considered. This behavior is observed for all the different MW frequencies studied. When we introduced linearly polarized radiation, $\rho_{x x}$ is almost the same if it is linearly polarized in the $\mathrm{x}$ or $\mathrm{y}$ direction. According to our model, the $\rho_{x x}$ response under MW excitation is governed by the term $A_{\mp} \cos w \tau: \rho_{x x} \propto A_{\mp} \cos w \tau$, where the amplitude $A_{\mp}$ has been defined above (see eq. (11)). Therefore for left and right circularly polarized radiation we would expect different results since the amplitudes are different. However if the damping factor $\gamma$ is larger than the MW frequency, $\gamma>w, \gamma$ would become the leading term in the corresponding denominator of the amplitude $A_{\mp}$. In this situation, $\gamma$ is able to quench the influence of the other terms and similar values are obtained for the amplitude of the orbit center for different polarizations. The same argument can be applied to both linearly and circularly polarized MW radiation. For GaAs, a value for $\gamma$ about $3 \times 10^{12} s^{-1}[23]$, is enough to obtain a similar $\rho_{x x}$ response irrespective of the specific MW polarization.

In conclusion, we have theoretically studied the influence of the microwave polarization on the magnetoresistivity response of two-dimensional electron systems. Linear and circular polarization have been considered with different senses. In agreement with previous experimental results, we show that, under strong enough damping, the $\rho_{x x}$ response is unaffected by changing the MW polarization.

This work has been supported by the MCYT (Spain) under grant MAT2005-06444 (JI and GP), by the Ramón y Cajal program (J.I.) by the EU Human Potential Programme: HPRN-CT-2000-00144.

[1] M.A. Zudov, R.R. Du, J.A. Simmons and J.L. Reno, Phys. Rev. B 64, 201311 (2001). 
[2] S.A. Studenikin, M. Potemski, A. Sachrajda, M. Hilke, L.N. Pfeiffer, K.W. West, Phys. Rev. B, 71, 245313, (2005).; S.A. Studenikin, M. Potemski, P.T. Coleridge, A. Sachrajda, Z.R. Wasilewski, Solid State Comm 129, 341 (2004).

[3] R.G. Mani, J.H. Smet, K. von Klitzing, V. Narayanamurti, W.B. Johnson, V. Umansky, Nature 420646 (2002).

[4] M.A. Zudov, R.R. Du, N. Pfeiffer, K.W. West, Phys. Rev. Lett. 90046807 (2003).

[5] R.L. Willett, L.N. Pfeiffer and K.W. West, Phys. Rev. Lett. 93026804 (2004).

[6] R.G. Mani, V. Narayanamurti, K. von Klitzing, J.H. Smet, W.B. Johnson, V. Umansky, Phys. Rev. B 69 161306(R) (2004); R.G. Mani, Appl. Phys. Lett. 85, 4962, (2004); R.G. Mani, Physica E, 22, 1, (2004).

[7] M.A. Zudov, R.R. Du, N. Pfeiffer, K.W. West, Phys. B 9073041303 (2006).

[8] C.L. Yang, R.R. Du, L.N. Pfeiffer and K.W, West, 74, 045315, (2006).

[9] R.G. Mani, Phys. Rev. B, 72 075327, (2005)

[10] M.A. Zudov, R.R. Du, N. Pfeiffer, K.W. West, Phys. Rev. Lett. 96, 236804 (2006).

[11] J.H.Smet, B. Gorshunov, C.Jiang, L.Pfeiffer, K.West, V. Umansky, M. Dressel, R. Dressel, R. Meisels, F.Kuchar, and K.von Klitzing, Phys. Rev. Lett. 95, 116804 (2005).

[12] A.C. Durst, S. Sachdev, N. Read, S.M. Girvin, Phys. Rev. Lett.91 086803 (2003)

[13] C.Joas, J.Dietel and F. von Oppen, Phys. Rev. B 72 , 165323, (2005).

[14] X.L. Lei, S.Y. Liu, Phys. Rev. Lett.91, 226805 (2003);

[15] V. Ryzhii and V. Vyurkov, Phys. Rev. B 68165406 (2003); V. Ryzhii, Phys. Rev. B 68193402 (2003); V.Ryzhii and R. Suris, J. Phys: Cond. Mat. 15, 6855, (2003) ; Ryzhii et al, Sov. Phys. Semicond. 20, 1299, (1986).

[16] P.H. Rivera and P.A. Schulz, Phys. Rev. B 70075314
(2004)

[17] Junren Shi and X.C. Xie, Phys. Rev. Lett. 91, 086801 (2003).

[18] A.V. Andreev, I.L. Aleiner and A.J. Millis, Phys. Rev. Lett. 91, 056803 (2003)

[19] J. Iñarrea and G. Platero, Phys. Rev. Lett. 94016806 , (2005)

[20] I.A. Dimitriev, M.G. Vavilov, I.L. Aleiner, A.D. Mirlin, and D.G. Polyakov, Phys. Rev. B, 71, 115316, (2005)

[21] M. Torres and A. Kunold, Phys. Rew. B. 71, 115313, (2005)

[22] Assa Auerbach and G. Venketeswara, cond-mat/0612469

[23] J. Iñarrea and G. Platero, Phys. Rev. B 72193414 (2005)

[24] X.L. Lei, S.Y. Liu, Phys. Rev. B 72, 075345 (2005)

[25] J. Iñarrea and G. Platero, Appl. Phys. Lett. 89, 052109, (2006).

[26] Kang-Hun Ahn, J. Korean Phys. Soc., 47 (4), 666-672, (2005).

[27] J. Iñarrea and G. Platero, Appl. Phys. Lett. 89, 172114, (2006).

[28] X.L. Lei, Phys. Rev. B 73, 235322, (2006)

[29] J. Iñarrea and G. Platero, to be published.

[30] V. Fock, Z. Phys. 47, 466 (1928); C.G. Darwin, Proc. Cambridge Philos. Soc. 27, 86, (1930).

[31] B.K. Ridley. Quantum Processes in Semiconductors, 4th ed. Oxford University Press, (1993).

[32] T. Ando, A. Fowler and F. Stern, Rev. Mod. Phys.,54,(1982).

[33] Jordan Kyriakidis, M. Pioro-Ladriere, M.Ciorga, A.S. Sachrajda and P. Hawrylak, Phys. Rev. B. 66, 035320, (2002).

[34] B.K. Ridley, Rep. Prog. Phys. 54, 169-256, (1991); B.M. Ashkinadze, V. Voznyy, E. Linder, E. Cohen, Arza Ron and L.N. Pfeiffer, Phys. Rev. B, 64, 161306 (2001). 